\title{
The Importance and Training Skills of Flexible Training in Classical Dance Teaching
}

\author{
Chao Qu \\ Taishan University, Tai'an, 271000, China
}

Keywords: Classical dance; History and culture; Modern dance. Training methods; Flexible training

\begin{abstract}
It cannot be divided that the form of classical dance with the country's long history. Compared with modern dance, classical dance has a more rigorous program as well as the relatively fixed action, skills. In classical dance, flexibility is of great significance to improve dancers dancing. Good flexibility training can not only strengthen the action of beautiful, also can improve posture. And at the same time, once the flexibility of the dancers appeared problem, it does not only affect the overall effect of stage performance, also the dancers themselves adverse impact on the body, such as muscle. So, it is help the people engaged in dance learning flexibility training requirements are put forward. In this paper, the classical dance of flexibility training study, analysis of flexibility training must value of existence and the specific training methods.
\end{abstract}

\section{Introduction}

The key of classical dance teaching is to dancer for expression of master and grasp rhythm, the flexibility of the teaching course of the body with high requirements. Only the flexibility to reach a certain level of the body can show a lasting appeal and its characteristics of classical dance, if there is not any high flexibility, so dancers graceful dance again also could not reflect the classical charm and elegance. Therefore, classical dance teaching, the key is to flexibility training dancers, avoid appearing "worse dancing" phenomenon in the process of dancing. In addition, the most important is the methods of flexible training to master, and avoided in the process of training muscle damage, etc., classical dance teacher can let the dancers in the flexibility of training to do some basic action and preparation, twisting his ankle and waist, prevent the damage without any preparation. Only scientific and reasonable training flexibility, so as to realize the lasting appeal of classical dance teaching, we can fundamentally get good effect in the teaching of classical dance.

\section{The importance of flexible training in classical dance teaching}

Germination and formation period of dance can be traced back to the conceptions of human development, beyond the human memory. Chinese classical dance as an important part of Chinese dance art, it is on the basis of the traditional folk dance, after generations of human carefully extraction, processing, sorting and created, follow the development of human beings and come down, it is model significance to the classical style of dance. With the development of The Times, dancing has become a specialized art. In today's classical dance has become a specialized course, teachers in the teaching process should give enough attention.

It is advantageous to learning behavioral essentials of the classical dance.

Compared with modern dance teaching, the teaching of classical dance, flexible training is very important. Students in the process of learning should master the basic behavioral essentials, namely: the lift, heavy, strong, rely on, ham, shy, move. Classical dance emphasizes the artistic conception, learners are required to pay attention to the combination of learning actions and thoughts, if learners in the learning process with no good flexibility training, that will not well combine artistic conception with the action of the degree. For example, some students begin to learn classical dance in their adult years, they are in the process of learning, they may have a very profound understanding of the essentials of dance, its body has begun at the forming stage, so the body is relatively hard, so in the learning activity, it cannot reach the standard which the classical dance moves need to reach, this part 
is very easy to hurt the enthusiasm of students to learn classical dance, the students to learn classical dance blowing to this part of the enthusiasm, in the end it is very easy to give up the follow-up study of dance., the teacher usually requires students can install complete action while classical dance teaching. If students had good flexibility training, students can be very easy when do actions completed quickly, for the adagio movement can also be round, very easy to meet the requirements of teachers, learning well will be very easy.

\section{Make the foundation of classical dance speed quality for the students}

Classical dance speed quality and speed of other dance quality requirements the same, the concrete content includes the following aspects: first of all, requires learners thinking reaction speed and movement speed very fast, which can quickly know when students learn the meaning of teachers, quickly grasp the behavioral essentials; Second, the learner's actions have to be very fast. Requires learners can in a short period of time, quickly complete the teachers' to a dance movements; finally, the learner movement speed is very fast. This refers to learners in learning dance moves, for quickly moving. Therefore, learners are learning classical dance, speed quality is very important. In the specific classical dance performances, the learner's speed quality will not only embody in the above several aspects, at the same time it also can reflect on the learners' reaction speed of dance music. A good speed quality of the dancer, sometimes his movement even than thinking reaction faster, and this is the result of long flexible training and rendering, thus it can be seen that flexible training be a very solid foundation for the study of the classical dance speed quality basis.

\section{It can help to improve the quality of teachers' teaching.}

In the teaching of classical dance, teachers is not hard to find, a lot of good students, they are not particularly sensitive to music, to classical knowledge accumulation may also is not very rich, but they can learn classical dance very well, this is mainly due to their good flexibility, the advantages of indirect makes up the defect of the music and the professional knowledge, to make this part of the students in the process of classical dance learning can still be very outstanding achievements, and gradually fall in love with dance learning. In addition, the classical dance teaching, the development of course is a very important part of a classical dance courses, but in the development of classroom teaching, flexible training is still the core content of students' training. Therefore, flexible training for classical dance teaching is significant.

From the above content analysis, it can be concluded that, for a dance teacher, if students study classical dance ready flexibility training, the teachers in the teaching process will have more time for students' deficiency of targeted training, according to the actual situation of the class, teachers can use these time designing different teaching law, according to their aptitude of dance teaching method and content of the training, they can make the teaching more interesting, active classroom atmosphere, to enhance teachers' teaching quality, improve the classroom efficiency.

\section{The training techniques of flexible training in the teaching of classical dance}

After a long period of classical dance teaching experience, it is concluded some training tips of classical dance flexible teaching:

\section{In front of the flexible training, we should do the corresponding preparation}

Classical dance for requires a high degree of flexibility, many action must have a good body flexibility to complete, but each person's physical condition is different, different individuals between body flexibility is put in bigger difference, it will be before the flexible training, need to be prepared for the corresponding, and the right training method, like this only then can achieve the desired training effect. Generally refers to after the deep breathing to make abdominal, starting from the head, to the end of the foot, simple activities open to the whole body muscle, it also avoids injury in training. Preparations must be done before training, it is advisable to about ten minutes or so, time is too short, the body's muscles not wake up, if the time is too long, it will consume too much energy, it is not conducive to the back of the flexible training. 


\section{Pay attention to the training volume, appropriate circuit training}

In flexible training, it can't let students excessive pursuit of explosive force, excessive consumption of energy, in the early stage, a small amount of training, and then based on the training of students' progress, gradually increase the volume, of course also should bear according to students' individual body ability to treat respectively. Flexibility and circularity, avoid a training practice, it has not been reduced flexibility. In flexibility training, must avoid fast action frequency phenomenon, adopts medium or slow motion frequency. Because of slow motion frequency medium can prolong the action time of force the joints, prevent ligament and muscle strain. At the time of use force to passive exercises, hard degree to increase gradually, and given priority to with the students' subjective feeling, when students appear muscle pain, it can try to insist on a minute, when students feel the muscle paralysis, we must stop practicing. In weight-bearing exercise, it is mainly to strengthen students' flexibility, not to exceed the negative weight elongated muscle strength can reach $50 \%$. Static tensile load can be appropriately increased some, dynamic swing motion, need appropriate to reduce some weight. Flexibility in general and in the training, all of which must ensure that the training volume and training intensity, moderate expansion circuit training, strengthen students' flexibility, step by step to achieve the desired training effect. At the same time, long-term change, regularization, systematic training, and gradually, gradually improve requirements, not to rush, to avoid injury.

\section{In flexibility training, pay attention to the combination of dynamic and static}

In flexibility training, pay attention to the combination of dynamic and static, but static time should not be too long, the best time is in about 10 seconds, static stretching after the body's muscles, pause a few seconds, then the dynamic training. In the practical training, it can according to the age of the students, the differences between the body shape, are carried out step by step, don't rush, so as to avoid injury.

\section{Avoid flexibility training only in a single location}

In flexibility training, it is not a joint or a body part, but all relevant parts of the integrated embodiment. "Driving axle" action, for example, it is mainly by the shoulders, in areas such as the spine and hip joint. Therefore, in practice the action time of the year, it needs to be trained in all parts of the flexibility, the part is bad, which will focus on training, achieve complement each other, then achieve the desired effect. In classical dance basic training, for example, " ZiJin jump" champions league for foot back, hip, shoulder, cervical vertebra, lumbar softness is better, and individual students due to the flexibility of the lumbar spine is poorer, the cause is stiff, not smooth, not stretch, to this, in the teaching, special training, if through the flexibility of the waist will greatly improve the quality of its actions. So, the whole body flexibility training, avoid by all means only training a certain parts of the body flexibility, this will make the body appears uncoordinated, jump out of the classical dance also will lose should have aesthetic feeling.

\section{The cautions of flexibility training}

For training, the students once found himself the body appear problem, they should immediately stop training, explain to the teacher and find out the reason, change the way of training or volume, until the body can adapt to. At the same time, in flexibility training, the teacher must remind students to pay attention to the development of each part, to ensure the students' body quality meet the demand of dance skills. In practical training, the teacher must be clear reversibility principle, namely flexibility training stop lead to declining flexibility or recovery. Therefore, they need to develop a training program for a long time, that will overcome difficulties, adhere to the flexibility training, guarantee the students' flexibility, and meet the demand of dance skills, effective meaning interpretation dance. Right flexibility training, it can make students muscles, ligaments, tendons and other stretching increases greatly, while training students can also avoid the body damage.

\section{Conclusion}

There is an old saying called: "a minute on stage, the audience ten years of work", this sentence described the flexible teaching in the dance is very appropriate. Flexibility for a dancer's requirement 
is very high, the dancers each part of the body have to be very flexible, it can undertake various high difficulty action, dancer's eye, the shoulders and hips should be flexible. Therefore, dancing teaching flexibility training is very important. The flexibility of the scientific teaching to cultivate dancers are very important. First of all, analyze and change the teaching methods, with teachers' teaching goal should not give priority to with fast, but according to their aptitude, according to each student's physical quality and dance talent for a coach, for the body supple dancer in guarantee the body from damage, increase to the training of the action, and improve the quality of the action, secondly, for the flexible students with poor performance of its basic counseling, guarantee flexibility can be more and more soft. At the same time, the cultivation of the flexibility can't depend on teachers and students in the flexibility of the trainer should preserve, flexible training is a long-term process, therefore, the teachers and students must work together.

\section{Reference}

[1] He Yinqiang. The importance and training technique of flexible training in classical dance teaching. Journal of education, 2014, (10): 164-164.

[2] Liu Ying. Introduction to flexible training in teaching of classical dance. Journal of literary life, literature and art theory, 2013, (7) : 126-126.

[3] Deng Xiaojing. Introduction to Chinese classical dance "firm" with "soft" training - introduction to the flexible quality of Chinese classical dance and the relationship between the explosive force training and teaching method. Journal of golden hill, 2012, (7): 94,78.

[4] Haimidan.Julaiti The importance of Chinese classical dance role in the Xin Jiang uygur dance. Journal of popular literature and art, 2013, (19) : 177. 